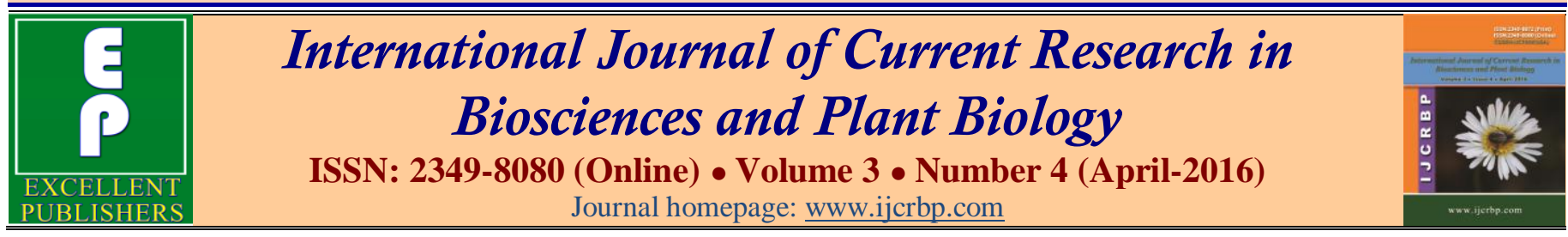

\title{
Analysis of Phospholipids Using the High Performance Liquid Chromatography Technique
}

\author{
Manoj G. Tyagi* \\ Department of Pharmacology and Medicinal Chemistry, Christian Medical College and Vellore Institute of Technology, Vellore- \\ 632 Oo2, Tamil Nadu, India \\ *Corresponding author.
}

\section{A bstract}

Phospholipids are precursors of molecules with many distinct roles ranging from structural to signaling aspects of the cell. Phospholipids belong to Zwitterionic molecules. They ionize in both positive and negative mode. Alterations in particular lipid levels change cellular behavior. In humans, these changes may manifest as diseases such as the Alzheimer's, atherosclerosis, diabetes, obesity, and even cancer formation. This has led to an enhanced interest in evaluating lipid changes both during normal cellular processes and in disease states. Along with the advent of rapid mass spectrometry, it has become possible to analyze many lipids within a single sample with unsurpassed sensitivity and detail. Coupling this with liquid chromatography potentially enables the complete profiling of all the phospholipids, both major and minor, from a single sample during a single analytical run.

\section{Article Info}

Accepted: 12 March 2016

Available Online: 06 April 2016

\section{Ke ywords}

Chromatography

Detector

HPLC

Phosphatidylcholine

Phosphatidylethanolamine

Phospholipids

\section{Introduction}

Phospholipids constitute complex and numerous groups of organic compounds occurring in the all living organisms as the essential components of cellular membranes i.e., lipid bilayer (Gładkowski et al., 2011). Phospholipids play significant roles in signaling mechanisms for cell proliferation and cell death (Gładkowski et al., 2012). Moreover, phospholipid metabolism plays an important role in numerous human diseases, cardiovascular, and cancer diseases, Alzheimer's and Parkinson's diseases and hyperglycemic syndromes. Food materials like the milk and Egg yolk included several classes of Phospholipids: phosphatidylcholine (PC), phosphatidylethanolamine (PE), sphingomyelin (SM), lysophosphatidylcholine (LPC), lysophosphatidylcholine (LPE), and phosphatidylglycerol (PG). The most important phospholipid fractions of egg yolk are PC and PE.
Phospholipids are analyzed by chromatographic techniques, such as the following: thin layer chromatography (TLC), gas chromatography (GC) (Anton, 2007). HPLC is characterized by high sensitivity and specificity, good resolution and high-speed process. Phospholipids are determined by reversed-phase liquid chromatography (RP-LC), normal-phase liquid chromatography (NP-LC), hydrophilic interaction liquid chromatography (HILIC), and multidimensional liquid chromatography (MDLC) (Sato et al., 2010; Zhao et al., 2011; Lima, 2002; Mazzella et al., 2004).

There are various detectors available for use on conjunction with liquid chromatography. Commonly, natural lipids are monitored by low-wavelength UV detectors and refractive index detectors (Romans and Palmer, 1972). However, UV response is highly dependent on the nature of fatty acid residues and varies with the degree of unsaturation within the same PL 
classes. Therefore, UV detection is not well adapted to the quantitative analysis of lipids with varying composition.

From this reason, very popular are aerosol detectors, such as such as evaporative light-scattering detectors (ELDS), and charged aerosol detectors (CAD, Corona). This study was carried out to determine the content of phosphatidylcholine and phosphatidylthanolamine in the egg yolk, goat milk and blood cells using the HPLC method and evaporative light scattering detector.

\section{Materials and methods}

\section{Reagents}

Standard compounds of L- $\alpha$-phosphatidylcholine (PC), L- $\alpha$-phosphatidylethanolamine $\quad(\mathrm{PE}), \quad$ L- $\alpha$-glycerophosphocholine (GPC) were purchased from Avanti Polar Lipid Co., USA and Sigma chemical co. The methanol for HPLC was obtained from Sigma-Aldrich (India).Water was purified using Milli-Q RG apparatus (Millipore USA) in our laboratory.

\section{Preparation of erythrocytes and extraction of lipids}

The technique used was based on described methods (Clemens and Waller, 1987). Goat blood was drawn into tubes containing $2 \mathrm{Na}$ EDTA. The blood was cooled in an ice bath and was kept in a refrigerator, and it was processed within a duration of $48 \mathrm{hrs}$. Plasma and buffy coat were removed after centrifugation at $1,000 \mathrm{~g}$ for 6 minutes at $4^{\circ} \mathrm{C}$, and the erythrocytes were washed three times in cold isotonic saline and run again at $1,000 \mathrm{~g}$ for 6 minutes at $4^{\circ} \mathrm{C}$. A small portion of the top layer was removed at each washing. Extraction of lipids from erythrocytes was essentially performed according to the method reported by Mawatari et al. (2009) and Mawatari and Murakami (1998).

\section{Solid phase extraction (SPE) of phospholipids}

This procedure basically involves a polar analyte, a midto non-polar sample solvent and a polar stationary phase. Retention of phospholipids, under normal phase conditions, is primarily due to the interactions between polar functional groups of phospholipid and polar groups on the sorbent surface. Non polar compounds are eluted in the early step of the procedure, whereas phospholipids interact with the polar groups on the sorbent surface. The disruption of this binding mechanism, i.e., the elution of phospholipids, is obtained by applying a solvent that is more polar than that in which sample is dissolved. Normal phase SPE with silica sorbent provided the most accurate results, except for Caboni et al. (1996) who had shown the high yield by using the C8 SPE .

\section{Extraction of phospholipids from egg yolk}

In this procedure, the off-line system of two dimensional liquid chromatography is applied. In first column, the preliminary separation of analyzed mixture at individual fraction was performed. The fractions were collected to eppendorf tubes and then the solvent was evaporated and again it was injected into second column. RP-HPLC method has been applied for the analysis of lipids in the first dimension using C18 column and methanol as a mobile phase (Caboni et al., 1996).

\section{HPLC analysis of phospholipids}

The HPLC analysis of phospholipids was performed using a Waters System (Alliance HPLC System 2695 separation module) coupled to a evaporative light scattering detector, data acquisitions and analysis were performed with a computer using the software version (Waters, India). Light scattering detectors detect phospholipids more accurately (Uran et al., 2001). Separation was carried out on an Extrasil silica $(150 \times$ $4.0 \mathrm{~mm}$ I.D., $3 \mu \mathrm{m}$ particle size) with a pre-column $(2 \times 4.0 \mathrm{~mm})$ from Phenomenex (USA). Phospholipids were separated by chromatography with isocratic elution with isopropanol-hexane-water $(53: 38: 9, \mathrm{v} / \mathrm{v} / \mathrm{v})$. The flow rate of the eluent was $1 \mathrm{ml} / \mathrm{min}$ and the column temperature was $35^{\circ} \mathrm{C}$. The volume of sample injected was $20 \mu \mathrm{l}$. The standard curves were developed from phospholipid concentrations of $0,200,400,600$, and $1000 \mu \mathrm{M}$ prepared in human plasma divided into $1 \mathrm{~mL}$ aliquots and stored frozen at $-70^{\circ} \mathrm{C}$ for less than 2 days. The linearity was determined by linear regression of phospholipid concentration on the basis of peak area ratios of phospholipids to the internal standard. The temperature of the detector was $80^{\circ} \mathrm{C}$ and the gas flow was $10 \mathrm{ml} / \mathrm{min}$. Compounds were identified by comparing the retention times of the sample peaks with those of the phospholipid standards.

\section{Statistical analysis}

All experiments were carried out five times and each experiments was analysed two times. Experimental data were treated by analysis of variance (ANOVA) using the statistical software SAS (SAS Institute Inc, Cary, NC, USA). 


\section{Results and discussion}

The phospholipids, phosphatidylcholine (PC) and phosphatidylethanolamine (PE) are important class of lipids. PC is a ley substrate for both the phospholipase D and $\mathrm{C}$ enzymes i.e., PC-PLC and PC-PLD. The breakdown of the membrane phospholipids by the phospholipases A2, B, C and D generates second messengers which bring about various biological changes mediated by hormones, drugs and toxins (Wagner et al., 1994). Thus it is imperative to study the content of phospholipids in the food as well as in the body fluids. The phospholipids are broadly classified into Glycerophospholipids and sphingolipids which are the two main groups belonging to the class of phospholipids. Glycerophospholipids are formed by glycerol, phosphoric acid, fatty acids and a hydroxy compound (e.g. choline, ethanolamine, serine, inositol). The structure of the most important phospholipids of milk fat: phosphatidylcholine (PC), phosphatidylethanolamine (PE), phosphatidylinositol (PI) and phosphatidylserine (PS). The two fatty acids, mainly represented by unsaturated FAs, are esterified at the $s n-1$ and $s n-2$ positions of the glycerol backbone. Phosphatidylcholine is a component of lecithin. Lecithin has been isolated from egg yolk, due to the chemical composition, is more valuable than the lecithin of plant origin, since the composition contains up to $80 \%$ choline (Fiume, 2001). It is known that choline is a main source of a precursor of the neurotransmitter, acetylcholine (Zeisel, 2000). Choline plays a role in liver function, prevention of cancer, and ensures the proper functioning of brain and development of memory i.e., maintenance of learning memory performance. Pro-health properties of phospholipids are determined by the presence of omega3 and omega- 6 fatty acids. Fatty acids above 20 carbon atoms is not found in the phospholipids isolated from plants materials. Therefore, phospholipids isolated from the egg yolk may be more useful in biomedical formulations. In this technique ordinary HPLC equipment with a evaporating light scattering detector was used. The results of this study are shown in Table 1. This study was conducted using an isocratic flow comprising of isopropanol-hexane and water which is efficient mobile phase for the separation of lipids. The results were reliable and the peak elution time for the PE and $\mathrm{PC}$ were on an average between 3.7 to 4.3 minutes and 5.6 to 6.8 minutes respectively.

Table 1. Content of two main phospholipids in goat milk, blood cells and egg yolk. Values are expressed as percentage of total phospholipids.

\begin{tabular}{lll}
\hline Sample & \% of PE & \% of PC \\
\hline Goat Milk & $31.5 \pm 1.23$ & $28.6 \pm 1.02$ \\
Goat blood cells & $24.3 \pm 0.89$ & $48.6 \pm 0.98$ \\
Egg yolk & $23.7 \pm 0.95$ & $32.9 \pm 1.07$ \\
\hline
\end{tabular}

In the human erythrocyte membrane, PS contains a higher amount of PUFA than PE, and PC contains a much lower amount of PUFA than PS and PE. It is known that phospholipids containing a high amount of PUFA are prone to oxidative stress. Actually, in previous reports it has been shown that relative composition of SM and PC increased in contrast to PS, PE, when human erythrocytes were incubated with tert -butyl hydroperoxide (tBHP). Therefore, the ratio of each glycerophospholipid to SM is a good indicator of the degree of peroxidative change of each glycerophospholipid. Apart from this, the pharmaceutical industry has implemented the use of synthetic phospholipids as drug carrier systems (Uran et al., 2001; Seri et al., 2010). This further necessitates analytical methods that have the ability to discriminate between endogenous and exogenous phospholipids in the biological system so pharmacokinetic and toxicokinetic data can be obtained. Phospholipids constitute $60 \%$ of the lipid mass of a eukaryotic cell membrane (Han and Gross, 2004), therefore, minute changes in phospholipids can lead to changes in the membrane that have major consequences on cell function and viability. The cell membrane has an asymmetrical distribution of phospholipids. Thus it can be concluded from this study that it is a specific and novel attempt to determine the two key phospholipids i.e., PC and PE from the goat milk, blood cells and egg yolk. The technique described is relatively cost effective and highly specific and can be utilised by both biomedical and food industry based laboratories.

\section{Conflict of interest statement}

Authors declare that they have no conflict of interest.

\section{References}

Anton, M., 2007. Composition and structure of hen egg yolk. In: Bioactive Egg Compounds (Eds.: Huopalathi, R., Lopez-Fandino, R., Anton, R., Schade, R.). Springer Verlag Berlin, Heidelberg. pp.1-6. 
Caboni, M.F., Menotta, S., Lercker, G., 1996. Separation and analysis of phospholipids in different foods with a lightscattering detector. J. Am. Oil Chem. Soc. 73, 1561-1566.

Clemens, M.R., Waller, H.D., 1987. Lipid peroxidation in erythrocytes. Chem. Phys. Lipids 45, 251-268.

Fiume, Z., 2001. Final report on the safety assessment of lecithin and hydrogenated lecithin. Int. J. Toxicol. 1, 2145.

Gładkowski, W., Chojnacka, A., Kiełbowicz, G., Trziszka, T., Wawrzeńczyk, C., 2012. Isolation of pure phospholipid fraction from egg yolk. J. Am. Oil Chem. Soc. 89, 179182.

Gładkowski, W., Kiełbowicz, G., Chojnacka, A., Gil, M., Trziszka, T., Dobrzański, Z., Wawrzeńczyk, C., 2011. Fatty acid composition of egg yolk phospholipids fractions following feed supplementation of Lohmann Brown hens with humic-fat preparations. Food Chem.126, 1013-1018.

Seri, H. I., Naim, H.Y., Hassan, T., 2010. A high performance liquid chromatography (HPLC) method with evaporative light scattering detector for quantification of major phospholipid classes of donkey serum Veternarski Archiv. 80(3), 365-373.

Han, X., Gross, R.W., 2004. Shotgun lipidomics: Electrospray ionization mass spectrometric analysis and quantitation of cellular lipidomes directly from crude extracts of biological samples. Mass Spectr. Rev. 24, 367-412.

Lima, A., 2002. High-performance liquid chromatography of fatty acids in biological samples. Anal. Chim. Acta 465, 81-91.

Mawatari, S., Murakami, K., 1998. Analysis of membrane phospholipid peroxidation by isocratic high-performance liquid chromatography with ultraviolet detection. Anal. Biochem. 264, 118-123.
Mawatari, S., Yunoki, K., Sugiyama, M., Fujino, T., 2009. Simultaneous preparation of purified plasmalogens and sphingomyelin in human erythrocytes with phospholipase A1 from Aspergillus orizae. Biosci. Biotechnol. Biochem. 73, 2621-2625.

Mazzella, N., Molinet, J., Syakti, A.D., Dodi, A., Doumenq, P., Artaud, J., Bertrand, J. C., 2004. Bacterial phospholipid molecular species analysis by ion-pair reversed-phase HPLC/ESI/MS. J. Lipid Res. 45(7), 13551363.

Romans, J. R., Palmer, I. S., 1972. Photoreflectometric method for the quantitative analysis of neutral lipids after thinlayer chromatography. Anal. Biochem. 49(2), 580-584.

Sato, Y., Nakamura, T., Aoshima, K., Oda, Y., 2010. Quantitative and wide ranging profiling of phospholipids in human plasma by two dimensional liquid chromatography/mass spectrometry. Anal. Chem. 82, 9858-9864.

Uran, A., Larsen, A., Jacobsen, P.B., Skotland, T., 2001. Species in human blood using normal-phase liquid chromatography coupled with electrospray ionization iontrap tandem mass spectrometry. J. Chromatogr. B. Biomed. Sci. Appl. 758, 265-275.

Wagner, B.A., Buetiner, G.R., Burns, C.P., 1994. Free radicalmediated lipid peroxidation in cells: oxidizability is a function of cell lipid bis-allylic hydrogen content. Biochem. 33, 4449-4453.

Zeisel, S. H., 2000. Choline: needed for normal development of memory. J. Am. Col. Nutr. 19, 528-553.

Zhao, Y-Y., Xiong, Y., Curtis, J.M., 2011. Measurement of phospholipids by hydrophilic interaction liquid chromatography coupled to tandem mass spectrometry: the determination of choline containing compounds in foods. J. Chromatogr. A. 1218, 5470-5479.

\section{How to cite this article:}

Tyagi, M. G., 2016. Analysis of phospholipids using the high performance liquid chromatography technique. Int. J. Curr. Res. Biosci. Plant Biol. 3(4), 28-39. doi: http://dx.doi.org/10.20546/ijcrbp.2016.304.005 